\title{
Toxoplasma gondii infection induces lipid metabolism alterations in the murine host
}

\author{
Ivan Milovanović1, Marija Vujanić², Ivana Klun², Branko Bobić², Aleksandra Nikolić², \\ Vladimir Ivović², Alexander M Trbovich¹, Olgica Djurković-Djaković ${ }^{2+}$
}

${ }^{1}$ Institute of Pathophysiology, School of Medicine, University of Belgrade, Belgrade, Serbia ${ }^{2}$ National Reference Laboratory for Toxoplasmosis, Serbian Centre for Parasitic Zoonoses, Institute for Medical Research, University of Belgrade, P.O. Box 102, 11129 Belgrade, Serbia

Host lipids have been implicated in the pathogenesis of Toxoplasma gondii infection. To determine if Toxoplasma infection influences the lipid status in the normal host, we assessed serum lipids of Swiss-Webster mice during infection with the BGD-1 strain (type-2) at a series of time points. Mice were bled at days zero and 42 post-infection, and subgroups were additionally bled on alternating weeks (model 1), or sacrificed at days zero, 14 and 42 (model 2) for the measurement of total cholesterol (Chl), high density lipoproteins (HDL), low density lipoproteins (LDL) and triglycerides and adiponectin. At day 42, brains were harvested for cyst enumeration. A significant decrease ( $p=$ $0.02)$ in HDL and total Chl was first noted in infected vs. control mice at day 14 and persisted to day $42(p=0.013)$. Conversely, $L D L$ was unaltered until day 42, when it increased ( $p=0.043)$. Serum LDL levels at day 42 correlated only with cyst counts of above 300 (found in $44 \%$ mice), while the change in HDL between days zero and 42 correlated with both the overall mean cyst count $(p=0.041)$ and cyst counts above $300(p=0.044)$. Calculated per cyst, this decrease in HDL in individual animals ranged from 0.1-17 $\mu \mathrm{mol} / \mathrm{L}$, with a mean of $2.43 \pm 4.14 \mu \mathrm{mol} / \mathrm{L}$. Serum adiponectin levels remained similar between infected and control mice throughout the experiment.

Key words: toxoplasmosis - murine infection - lipid metabolism - cholesterol - adiponectin

There is an emerging body of evidence showing that the intracellular protozoan Toxoplasma gondii alters host cell metabolism for entry and replication and uses host metabolic products for its own metabolic pathways. Toxoplasma cannot synthesize cholesterol (Chl) de novo and depends upon acquisition of low density lipoprotein (LDL)-derived Chl from the host cell, via endocytosis mediated by the LDL receptor (Coppens et al. 2000) or the LDL receptor-related protein (Portugal et al. 2008). A mechanism by which host and not parasite $\mathrm{Chl}$ controls the entry of Toxoplasma into cells has been proposed (Coppens \& Joiner 2003). These studies indicate that $\mathrm{Chl}$ does have an important role in pathogenesis of toxoplasmosis. However, data on parasite lipid sources are scarce and the molecular mechanisms by which Toxoplasma acquires host cell lipids are largely unknown (Coppens 2006).

To determine if Toxoplasma infection influences the host lipid status in vivo, serum lipid levels were assessed at different time points during infection in wild-type mice.

\section{MATERIAL AND METHODS}

Mice - Female Swiss-Webster mice (Medical Military Academy Animal Research Facility, Belgrade) weighing 18-20 $\mathrm{g}$ at the beginning of each experiment, were used. Mice were housed at six per cage and offered

Financial support: Ministry of Science and Technological Development of Serbia (145002)

+ Corresponding author: olgicadj@imi.bg.ac.yu

Received 10 October 2008

Accepted 17 February 2009 regular mouse feed and drinking water ad libitum. The study protocol was approved by the Institute for Medical Research Ethics Committee.

Parasites - The low virulence BGD-1 strain (human origin type-2 strain) characterized in our laboratory (Djurković-Djaković et al. 2006) was used for experimental infections. Mice were inoculated by oropharyngeal gavage with eight cysts, an inoculum shown to be non lethal for $>90 \%$ of the mice during the first eight weeks post-infection (p.i.).

Experimental design - Two different experimental models were used. To examine the kinetics of the lipids at an individual level during Toxoplasma infection (model 1), animals were infected $(\mathrm{n}=54)$ or left uninfected $(n=18)$ to serve as controls. Both groups were bled at days zero and 42 p.i. and subgroups of 6-12 mice were bled on alternating weeks at days 7, 14, 21 and 28, for the measurement of total $\mathrm{Chl}$, high density lipoprotein (HDL), LDL and triglyceride levels. At day 42 p.i., mice were euthanized and their brains were harvested for cyst enumeration. The experiment was performed twice and the data shown are their cumulative results.

Model 2 was designed to correlate blood lipid levels with adiponectin as well as with local changes in tissues. Groups of infected $(n \geq 9)$ and control $(n=6)$ mice were euthanized at days zero, 14 and 42 p.i. and blood was drawn for the measurement of lipids as above and of adiponectin. Brains and livers were harvested for future tissue and molecular analyses.

Lipids - Total Chl, HDL, LDL and triglycerides were measured on the Olympus AU 400 biochemical analyzer, according to the manufacturer's recommendations. 
Adiponectin - Adiponectin levels were determined using a sandwich enzyme immunoassay test kit (Quantikine Mouse Adiponectin/Acrp30 Immunoassay, R\&D Systems, Abingdon, UK) according to the manufacturer's instructions. The optical density was read on a Multiscan (Labsystems, Helsinki, Finland) microplate reader. The assay was performed in duplicate and mean values were calculated using a standard curve as described by the manufacturer. The sensitivity threshold was $0.003 \mathrm{ng} / \mathrm{mL}$.

Cyst enumeration - Immediately after euthanasia, brains were harvested and homogenized in PBS. For cyst enumeration, $25 \mu \mathrm{L}$ of the brain suspensions was placed on slides and cysts were counted under a phase-contrast microscope (Axioskop 2 Plus Zeiss). The number of cysts per brain was calculated by multiplying the number counted in four drops (by 3 experienced investigators) by 10 , giving a sensitivity threshold for our method of 10 cysts per brain.

Statistics - All statistical analyses were performed using the SPSS (version 10.0) package (SPSS Inc, Chicago, IL, USA). Differences between Chl fraction means within particular groups at different time points were evaluated by ANOVA, followed by Fischer's LSD test, while differences in Chl fraction means and adiponectin levels between infected and control group means were analyzed by the Student $t$-test or the Mann-Whitney test, as appropriate. Correlation between cyst number and Chl fractions was determined by Spearman's rank coefficient and correlation between cyst number and change in Chl fractions from day zero to day $42(\Delta)$ by two-tailed Pearson's coefficient. The level of significance was 0.05 .

\section{RESULTS}

The kinetics of serum lipids in mice during Toxoplasma infection is presented in Fig. 1. No difference between infected and control animals was seen at day 7 p.i.; however, at day 14, the mean Chl level decreased sharply $(p=0.019)$ and although slightly increasing thereafter, remained significantly $(p=0.045)$ decreased at day 42 as compared to control levels (Fig. 1A). This decrease in Chl was reflected in the same kinetics in HDL levels; i.e. abrupt decrease at day $14(\mathrm{p}=0.02)$ and lower HDL levels at day 42 as compared to controls $(p=0.013)$ (Fig. 1B). Conversely, LDL levels were comparable between infected and control animals by day 28 , including an unexpected rise at day 21 vs. previous time points (Fig. 1C). Only at day 42 was a small, albeit significant $(p=0.043)$ increase in LDL observed in infected animals. Some changes in triglyceride levels were observed during the experiment, but were seen in both infected and control mice and thus resulted in no significant alterations at any time point (Fig. 1D).

The cyst numbers recovered at day 42 varied greatly (mean $\pm \mathrm{SD}=916 \pm 1677$, range 20-7460 per brain); how-
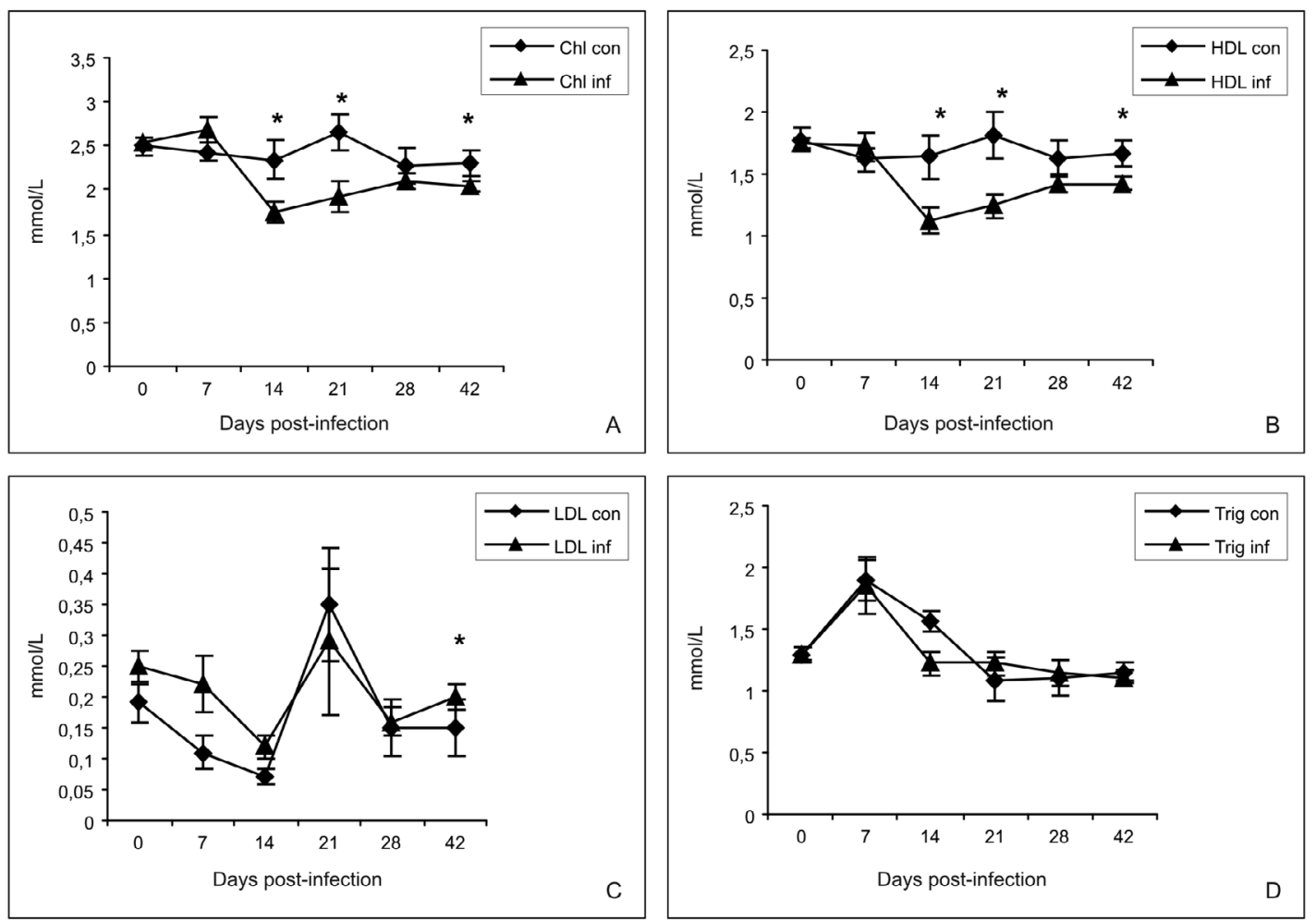

Fig. 1: kinetics of serum lipid levels (mean $\pm \mathrm{SD}$ ) during the course of Toxoplasma infection in Swiss Webster mice induced with eight cysts of the BGD-1 strain. Groups of mice $(\mathrm{n}=6-12)$ bled at alternative weeks and euthanized at day $42(\mathrm{n}=54)$. A: total cholesterol $(\mathrm{Chl})$ in infected (Chl inf) and control (Chl con) mice; B: high density lipoprotein (HDL) in infected (HDL inf) and control (HDL con); C: low density lipoprotein (LDL) in infected (LDL inf) and control (LDL con); D: triglycerides (Trig) in infected (Trig inf) and control (Trig con). Asterisk: $\mathrm{p}<0.05 \mathrm{vs.} \mathrm{control} \mathrm{mice.}$ 


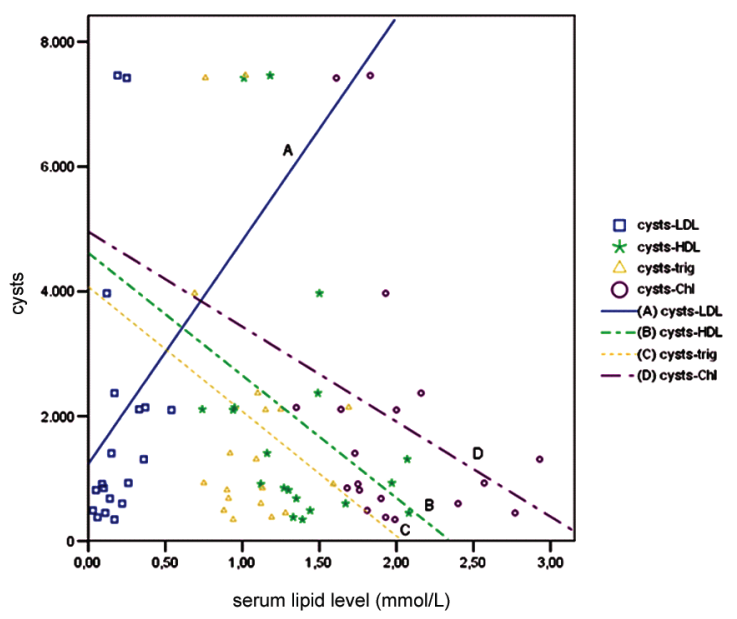

Fig. 2: correlation between serum lipid levels by fraction and Toxoplasma brain cyst counts above 300 harvested at day 42 post-infection (Spearman's rank coefficient).

ever, notably, only three animals had cyst counts above 2,500. Correlation analyses of the cyst counts at different levels with all lipid fractions (Fig. 2) revealed only one significant result - a positive correlation (Spearman's $r=$ $0.517, p=0.023$ ) between cyst counts above 300 (found in $44 \%$ mice) and LDL level. More importantly, however, when the cyst counts in individual animals were correlated with the change in Chl fractions from $\mathrm{d} 0-\mathrm{d} 42(\Delta)$, a positive correlation (Pearson) was obtained between $\Delta$ HDL and both the overall mean cyst count $\left(\mathrm{r}^{2}=1, \mathrm{p}=\right.$ $0.041)\left(\right.$ Fig. 3A) and cyst count above $300\left(r^{2}=0.22, p=\right.$ 0.044 ) (Fig. 3B). The decrease in the level of HDL per cyst in individual animals ranged from $0.1-17 \mu \mathrm{mol} / \mathrm{L}$ (possibly reflecting variation in cyst size and/or content), with a mean of $2.43 \pm 4.14 \mu \mathrm{mol} / \mathrm{L}$. No correlations between $\Delta$ LDL and either overall cyst count $\left(\mathrm{r}^{2}=0.05, \mathrm{p}=0.162\right)$ or cyst count above $300\left(\mathrm{r}^{2}=0.17, \mathrm{p}=0.081\right)$ were found.

In model 2, lipid levels in infected vs. control mice showed a trend similar to that in model 1, i.e., a decrease in HDL at day 14 and comparable levels of triglycerides, but the increase in LDL noted at day 42 did not reach significance (due to a lower number of animals per group and hence a lower statistical power). No significant differences in adiponectin levels were found between infected and control groups at either days 14 or 42 .

\section{DISCUSSION}

Chl metabolism in mammalian cells involves direct transport of preformed sterol from the liver to the periphery, in the form of VLDL and LDL. On the other hand, tissue $\mathrm{Chl}$ is transported from peripheral cells through the plasma compartment back to the liver in the form of HDL (Fielding \& Fielding 1995).

The major lipid metabolism alterations obtained in a model of experimental toxoplasmosis in normal mice included a decrease in serum HDL and, accordingly, in total Chl, beginning at day 14 p.i. and continuing until the end of the experiment at day 42; an increase in LDL only at this latter time point; and no alterations in triglyceride

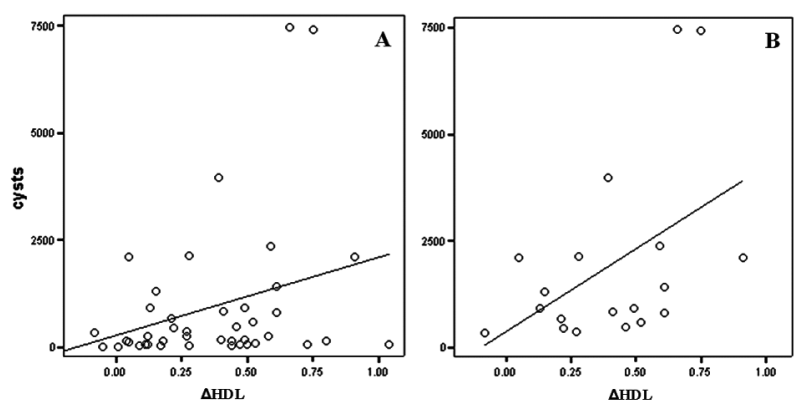

Fig. 3: correlation between changes from day 0-42 serum high density lipoprotein (HDL) in individual mice ( $\triangle \mathrm{HDL}$ ) and total counts (A) and counts above 300 (B) of Toxoplasma brain cysts harvested at day 42 post-infection (Pearson's correlation coefficient).

levels at any time point. Furthermore, the cyst counts positively correlated with the change between pre-infection and day 42 HDL levels, while only higher ( $>300)$ cyst counts correlated with day 42 serum LDL levels.

Lipid metabolism in parasitic infections is poorly understood. Data from experimental models are mainly derived from studies addressing the issue of the atherogenic potential of parasitic infection. Normal mice infected with Trypanosoma cruzi and Schistosoma mansoni were found to have decreased levels of total blood Chl. However, in the trypanosome infection model, Chl fractions have not been analyzed (Sunnemark et al. 2000). On the other hand, while $S$. mansoni is a trematode completely unrelated to Toxoplasma, schistosomes do not synthesize Chl, but incorporate host LDL via inducible LDL receptors. In $S$. mansoni infected outbred mice, a reduction in total $\mathrm{Chl}$ and HDL and an insignificant rise in LDL was shown at a time at which infection became patent (Doenhoff et al. 2002). This is quite similar to what we showed in our model of Toxoplasma infection. However, our results are difficult to compare with previous work, since the only available data are obtained from studies in spontaneously atherosclerotic mice. In two models of genetically modified mice, i.e. ApoE deficient mice with consequently high VLDL and total Chl levels (four-fold higher than in normal mice as calculated with a conversion coefficient 0.0259 for $\mathrm{mg} / \mathrm{dL}$ to $\mathrm{mmol} / \mathrm{L}$ ) and $\mathrm{LDL}$ receptor deficient mice (LDLr-/-) who have high levels of LDL and total Chl, Portugal et al. (2004, 2008) showed that infection with cysts of the Me49 strain (a typical type-2 strain) induced a decrease in total Chl and VLDL levels, while HDL levels remained unaltered. In the LDLr-/- mice, there was also a decrease in the LDL levels. The observed decrease in the initially high levels of VLDL and LDL was attributed to acquisition by Toxoplasma. This is logical, as LDL is the major lipoprotein from which Toxoplasma takes Chl (Coppens et al. 2000). However, in our model of Toxoplasma infection in normal mice we did not see a decrease in LDL levels. To the contrary, serum LDL levels were increased at day 42 , i.e., at a time of established chronic infection. To integrate our results with the above data, it should first be 
noted that, unlike ApoE- and LDLr-/- mice, normal mice have low serum LDL (Khovidhunkit et al. 2004) and high serum HDL (Lee \& Choudhury 2007) levels. Thus, it may be postulated that upon infection with Toxoplas$m a$, uptake of $\mathrm{Chl}$ from serum LDL by proliferating parasites triggers mobilization of $\mathrm{Chl}$ from the liver to the periphery. Due to the use of $\mathrm{Chl}$ in peripheral tissues by Toxoplasma, less remains to be returned to the liver and thus decreased serum HDL is observed. The decrease at day 14 (but not at day 7) coincides with the time when the parasite burden has increased sufficiently to require significant LDL acquisition, measurable (in a host with low LDL and high HDL) only by HDL decrease. Moreover, the lower levels of $\mathrm{Chl}$ returned to the liver in the form of HDL are further explained by the correlation between cyst counts and changes from the baseline to the day 42 levels of HDL. Similarly, the significance of LDL as a Chl source for Toxoplasma is emphasized by the correlation between higher brain cyst burdens and increased day 42 serum LDL levels, reflecting increased mobilization of $\mathrm{Chl}$ from the liver.

Another reason for decreased HDL may be the acute phase response, which is an immediate reaction, associated with hypertriglyceridemia. However, since the decrease in HDL levels in our model did not occur until day 14 , and persisted up to day 42 with no changes in triglycerides, the lipid alterations observed do not seem to be part of the acute phase response.

In our model, lipid alterations were not mediated by adiponectin. Adiponectin is a fat tissue hormone involved in lipid and carbohydrate metabolism, the circulating levels of which, under physiological conditions, positively correlate with plasma HDL concentrations (Cnop et al. 2003, Tschritter et al. 2003). We found no change in plasma adiponectin levels in infected animals, indicating no mediation by adiponectin of the HDL alterations observed. Hence, it appears that Toxoplasma induces serum lipoprotein changes by influence on lipid receptors and apolipoproteins. To clarify the mechanisms of lipid metabolism alterations during experimental toxoplasmosis, we are currently looking into $\mathrm{Chl}$ receptors and transport proteins at the tissue and molecular level.

The use of outbred animals in our study may have accounted for the great variation in cyst burden as well as for the variation in some lipid fractions among individual animals. An advantage of such a model, however, is that it examines lipid metabolism during the natural course of infection in the wild-type murine host. The lipid alterations observed despite individual variations only emphasize the burden of Toxoplasma infection on host lipid homeostasis, and homeostasis in general.

\section{ACKNOWLEDGEMENTS}

To the expert technical assistance of Mrs. Jordanka Djurović.

\section{REFERENCES}

Cnop M, Havel PJ, Utzschneider KM, Carr DB, Sinha MK, Boyko EJ, Retzlaff BM, Knopp RH, Brunzell JD, Kahn SE 2003. Relationship of adiponectin to body fat distribution, insulin sensitivity and plasma lipoproteins: evidence for independent roles of age and sex. Diabetologia 46: 459-469.

Coppens I 2006. Contribution of host lipids to Toxoplasma pathogenesis. Cell Microbiol 8: 1-9.

Coppens I, Joiner KA 2003. Host but not parasite cholesterol controls Toxoplasma cell entry by modulating organelle discharge. $\mathrm{Mol}$ Biol Cell 14: 3804-3820.

Coppens I, Sinai AP, Joiner KA 2000. Toxoplasma gondii exploits host low-density lipoprotein receptor-mediated endocytosis for cholesterol acquisition. J Cell Biol 149: 167-180.

Djurković-Djaković O, Klun I, Khan A, Nikolić A, Knežević-Ušaj S, Bobić B, Sibley LD 2006. A human origin type II strain of Toxoplasma gondii causing severe encephalitis in mice. Microbes Infect 8: 2206-2212.

Doenhoff MJ, Stanley RG, Griffiths K, Jackson CL 2002. An antiatherogenic effect of Schistosoma mansoni infections in mice associated with a parasite-induced lowering of blood total cholesterol. Parasitology 125: 415-421.

Fielding CJ, Fielding PE 1995. Molecular physiology of reverse cholesterol transport. J Lipid Res 36: 211-228.

Khovidhunkit W, Kim MS, Memon RA, Shingenaga JK, Moser AH, Feingold KR, Grunfeld C 2004. Effects of infection and inflammation on lipid and lipoprotein metabolism: mechanisms and consequences to the host. J Lipid Res 45: 1169-1186.

Lee JMS, Choudhury RP 2007. Prospects for atherosclerosis regression through increase in high-density lipoprotein and other emerging therapeutic targets. Heart 93: 559-564.

Portugal LR, Fernandes LR, Cesar GC, Santiago HC, Oliveira DR, Silva NM, Silva AA, Lannes-Vieira J, Arantes RME, Gazzinelli RT, Alvarez-Leite JI 2004. Infection with Toxoplasma gondii increases atherosclerotic lesion in ApoE-deficient mice. Infect Immun 72: 3571-3576.

Portugal LR, Fernandes LR, Pietra Pedroso VS, Santiago HC, Gazzinelli RT, Alvarez-Leite JI 2008. Influence of low-density lipoprotein (LDL) receptor on lipid composition, inflammation and parasitism during Toxoplasma gondii infection. Microbes Infect 10: $276-284$.

Sunnemark D, Harris RA, Frostegård J, Örn A 2000. Induction of early atherosclerosis in CBA/J mice by combination of Trypanosoma cruzi infection and a high cholesterol diet. Atherosclerosis 153: 273-282.

Tschritter O, Fritsche A, Thamer C, Haap M, Shirkavand F, Rahe S, Staiger H, Maerker E, Häring H, Stumvoll M 2003. Plasma adiponectin concentrations predict insulin sensitivity of both glucose and lipid metabolism. Diabetes 52: 239-243. 